\title{
Role of Pregabalin to Decrease Postoperative Pain in Microdiscectomy: A Randomized Clinical Trial
}

Shahzaib R. Baloch ${ }^{1}$, Imtiaz A. Hashmi ${ }^{2}$, Mohammad S. Rafi ${ }^{3}$, Ambreen Wasim ${ }^{4}$, Saddam Mazar ${ }^{5}$, Nadia Malick $^{6}$, Banin Tayyab ${ }^{7}$, Hoordana Riaz ${ }^{8}$

1. Orthopaedics, Dr. Ziauddin Hospital, Karachi, PAK 2. Orthopedics/ Consultant Spine and Orthopedics Surgeon, Agha Khan University Hospital, Karachi, Karachi, PAK 3. Orthopedics Department/ Spine and Orthopedic Surgeon, Dr. Ziauddin Hospital, Karachi, PAK 4. Department of Research , Ziauddin University, Karachi, PAK 5. Orthopaedic Surgery, Dr. Ziauddin Hospital, Karachi, PAK 6. Department of Internal Medicine, Dr. Ziauddin Hospital, Karachi, PAK 7. Orthopedics/Intern, Dr. Ziauddin Hospital, Karachi, PAK 8. General Surgery, Bolan Medical Complex Hospital, Quetta, PAK

Corresponding author: Shahzaib R. Baloch, sheekdurrazai@gmail.com

\section{Abstract \\ Purpose}

The purpose of this study is to compare the effect of pregabalin in reducing the neuropathic pain in postoperative patients who have undergone single-level microdiscectomy for prolapsed intervertebral lumbar disc.

\section{Methods}

A randomized control clinical trial was conducted from June 2018 to April 2020 in three campuses Dr. Ziauddin University Hospital, Karachi, by two spinal surgeons. This study included 84 patients who underwent either emergency or elective microdiscectomy surgery. The patients randomized into two equal groups of 42, (group-A: pregabalin) and (group-B: placebo). Both groups also received routine analgesia along with the pregabalin and placebo capsules. In the intervention group, pregabalin was administered preoperative and postoperative defined times. The pain scores were recorded by visual analog scale (VAS) and Roland-Morris score system on the preoperative day and compared to the scores on follow-up on postoperative day seven.

\section{Results}

The pain scores were significantly better in group-A compared to group-B with similar baseline variables. The mean VAS scores of pains in group-A on postoperative day seven on follow-up were compared to VAS pain scores in group-B showing better pain control. The Roland-Morris scores were also significantly better for group-A.

Review began 01/05/2021 Review ended 01/22/2021 Published 01/22/2021

\section{(๑) Copyright 2021}

Baloch et al. This is an open access article distributed under the terms of the Creative Commons Attribution License CC-BY 4.0., which permits unrestricted use, distribution, and reproduction in any medium, provided the original author and source are credited.

\section{Conclusions}

The use of pregabalin in addition to the routine analgesia has better control of postoperative neuropathic pain in patients with single-level microdiscectomy compared to the patients who are receiving only routine analgesia. Other factors like cost, dose, side effects, and frequency should also be considered.

Categories: Pain Management, Neurosurgery, Orthopedics

Keywords: prolapsed intervertebral disc, microdiscectomy, pregabalin, neuropathic pain, postoperative pain

\section{Introduction}

Lower back pain with severe radiculopathy is caused by the herniation of the lumbar disc into the spinal column with nerve root compression. This herniation can lead to devastating outcomes if not treated. In case of complications of untreated prolapsed discs like cauda equina syndrome, urgent surgical intervention is required. A minimally invasive approach to this surgery like "microdiscectomy" is necessary to relieve pain and to prevent further progression of damage.

Discectomy is a routine procedure performed by orthopedic surgeons and neurosurgeons, and it is associated with mild to moderate levels of postoperative pain. Multimodal treatments have been used to reduce side effects and doses to achieve adequate analgesic effects [1]. Treatment of postoperative pain poses significant challenges in postoperative care due to ethical, medical, and humanitarian reasons as proposed by Kehlat et al. [1] and it is thus crucial to manage it efficiently, using a multimodal approach for optimum recovery response, as endorsed by the American Society of Anesthesiologists (ASA) [2].

As the number of day-care procedures has increased by a significant number globally, newer, and more 
complex methods are being devised almost daily. While this may have its own benefits [3], these procedures may cause the patient to experience more pain in the initial postoperative period due to surgical trauma induced hyperalgesia [4]. Despite that, microdiscectomies are being performed in ambulatory settings on a regular basis. While initially, the standard method for preventing and treating acute postoperative pain was by administering opioid analgesics, it has, in recent times, been replaced by a combination of nonopioid analgesic drugs with different mechanisms of action as part of a multimodal approach to prevent pain $[5,6]$.

Almost $80 \%$ of patients still suffer from postoperative pain despite all the recent advances [7] which pose as a threat towards the road of recovery of the patient, both physically as well as mentally, and thus prolonging the time to recover and achieve milestones, whilst occasionally lengthening the hospital stay, thereby making the patient more vulnerable to acquiring opportunistic infections, amongst other postoperative complications [8].

It is often noted that the risk of inappropriate pain control in patients undergoing surgery, has been relatively higher in patients who are suffering from chronic back pain, as they have already developed a tolerance to strong analgesic [9]. One of the possible reasons for the poor response to analgesics could be due to the loss of opioid receptors at the site of compression/peripheral nerve pathology. However, increasing the dosage of the opioid has proven to show adequate pain control, although this may lead to tolerance and subsequent dependence on the drug, and a phenomenon known as opioid-induced hyperalgesia [9].

It has been noted that administration of epidural analgesia has shown promising results in significantly reducing postoperative pain after spinal surgeries [10]. It has also been proven, by Mathiesen et al, that a multimodal approach prevents the usage of opioids for postoperative pain management, thereby reducing the potential side effects and addictive properties of said agents [11], especially when the dosage of pregabalin is kept $>300 \mathrm{mg}$ as suggested by a meta-analysis carried out by Lam et al. [12].

When discussing the management of acute and/or chronic states of pain, such as diabetic peripheral neuropathy, gabapentin has not shown to have much significance over other agents, such as tricyclic antidepressants (amitriptyline), and thereby are often used alone or in combination depending on the condition of the patient and their comorbidities [13], however, pregabalin has shown to have a much superior pharmacokinetic profile than its precursor [14].

Pregabalin is structurally a gamma-aminobutyric acid (GABA) and has the same characteristics and mechanism of action as its precursor gabapentin [14]. For managing and treating acute pain, the efficacy of pregabalin is similar to that of gabapentin; however, having a greater bioavailability, linear pharmacokinetics, lower dose titration, and a shorter-acting time to achieve optimal analgesic effect makes pregabalin more superior to its precursor $[14,15]$. Pregabalin also appears to have a higher success rate for managing acute nociceptive pain postoperatively, and thereby reducing opioid-induced dependence and anxiety. $[14,16,17]$

Pregabalin has been administered as part of analgesic regimens for various procedures, including lumbar spine surgery, in a few studies. One such study conducted by Mohsin et al., revealed, the effectiveness of both agents (gabapentin and pregabalin), for postoperative management for single-level microdiscectomy was almost equivalent. [18]

In other studies, pregabalin was either compared to a placebo or there were limitations in the study design, with respect to the pharmacodynamics of the drug, such as it being utilized just before the surgery, instead of administering it at least a week prior in order to produced successful results, as seen in reality [14,19]. Thus, in order to achieve maximal benefits, interventional drugs should be administered one week prior to the actual procedure, as the preemptive use of GABA analogs preoperatively reduces not just the side effects, but the requirement for postoperative analgesia as well.

Therefore, we intended to compare the postoperative pain control in patients with the usage of low dose pregabalin along with routine analgesia to those with patients who received only routine analgesia pre and postoperatively.

We hypothesized that the pain in the pregabalin group would be less than that in the without pregabalin group.

\section{Materials And Methods}

This study is a single-blinded, parallel-group, randomized clinical trial that was conducted in the orthopedics department of Dr. Ziauddin University Hospital Karachi. Conducted on patients who were suffering from chronic sacral radiculopathy and diagnoses to have PID (prolapsed intervertebral disc), undergoing elective lumbar microdiscectomy. The study started with IRB (institutional review board) approval and written/verbal informed consent from the patients. Then they were randomly allotted in two groups: group-A (pregabalin group) and group-B (control group). Each group consisting of 42 patients each with a total of 84 patients, aged between 27 years to 61 years. The exclusion criteria included patients with 
previous lumbar surgery, patients allergic to gabapentinoids, patients with renal impairments, patients already on opioids, benzodiazepines, barbiturates, ethanol (alcohol), diabetes, and other drugs that depress the central nervous system and on angiotensin-converting enzyme (ACE) inhibitors as they may enhance the adverse/toxic effect of pregabalin.

In this study, the patients received pregabalin $75 \mathrm{mg}$ or placebo (sucrose) twice daily from preoperative day till one week of surgery. All medications were identical and provided by the hospital pharmacy in sealed opaque envelopes. Along with this, all patients were getting regular postoperative pain management with paracetamol 1 gram six hourly and nonsteroidal anti-inflammatory medication (Ketorolac) $30 \mathrm{mg} \mathrm{I} / \mathrm{V}$ ter die sumendum (TDS) as a primary protocol for pain management. Opioids were given on pro re nata (PRN) bases if the pain was not controlled via the primary protocol. All patients were given three pain scale questionnaires Roland-Morris disability scale and visual analog scale (VAS) pain to fill, preoperatively, and then after one week of surgery, and the results were compared.

Data was analyzed using Excel 2013 and IBM SPSS Statistics ver.20 (SPSS Inc., Chicago, IL, USA). Contingency tables were analyzed using Fisher exact test, $P$ values $<0.05$ were considered statistically significant.

\section{Results}

Our study comprised of 84 patients, equally divided into two groups based on random selection; group A consisted of 42 patients, who received pregabalin pre and postoperatively, and group B consisted of the remainder of patients who did not receive pregabalin. Amongst our participants, $58 \%(n=49)$ of participants were females, while the remaining $42 \%$ ( $n=35$ ) were males. The age of the participants ranged between 27 years to 61 years, categorizing them as adults, and thus all the participants received the standardized adult dosage of the analgesics (including pregabalin for group A only). For the assessment of pain, two international scoring systems; the 10-point VAS and the 24-item patient-reported Roland-Morris scoring system for lower back pain (RMS), were used. The pain was assessed both preoperatively and one week postoperatively, in both groups. Keeping the significance of $\mathrm{P}$ at $<0.05$, the results of our research are as follows:

When comparing the pre and post-operative VAS scores of group A, (Figure 1), $95.2 \%(\mathrm{n}=40)$ patients complained of experiencing severe pain preoperatively which significantly improved post-operatively after receiving treatment with pregabalin (along with other analgesic agents) and reduced to $2 \%$ of participants of group A $(n=1)$ experiencing moderate pain, while almost $81 \%$ of patients $(n=34)$ endured mild pain and nearly $16 \%(n=7)$ felt no pain (Figure 2). In contrast to that, just about $93 \%$ of patients $(n=39)$ in group B experienced severe pain preoperatively (Figure 1$)$, which reduced to only $16 \%(n=7)$ feeling moderate pain, nearly $79 \%(n=33)$ enduring mild pain and around $4 \%(n=2)$ of patients experiencing no pain (Figure 2). The preoperative VAS score for group A was 8.33, which significantly lowered to 1.31 postoperatively, seen in (Figure 3). Whereas (Figure 4) shows that the group not receiving pregabalin (group B), the preoperative VAS amounted to 7.98 which reduced to 2.50 postoperatively. The average VAS for group A totaled 7.02, while for group B it came to 5.48, (Figure 5). In either of the groups, no patients reported severe pain, postoperatively, regardless of the administration of pregabalin.

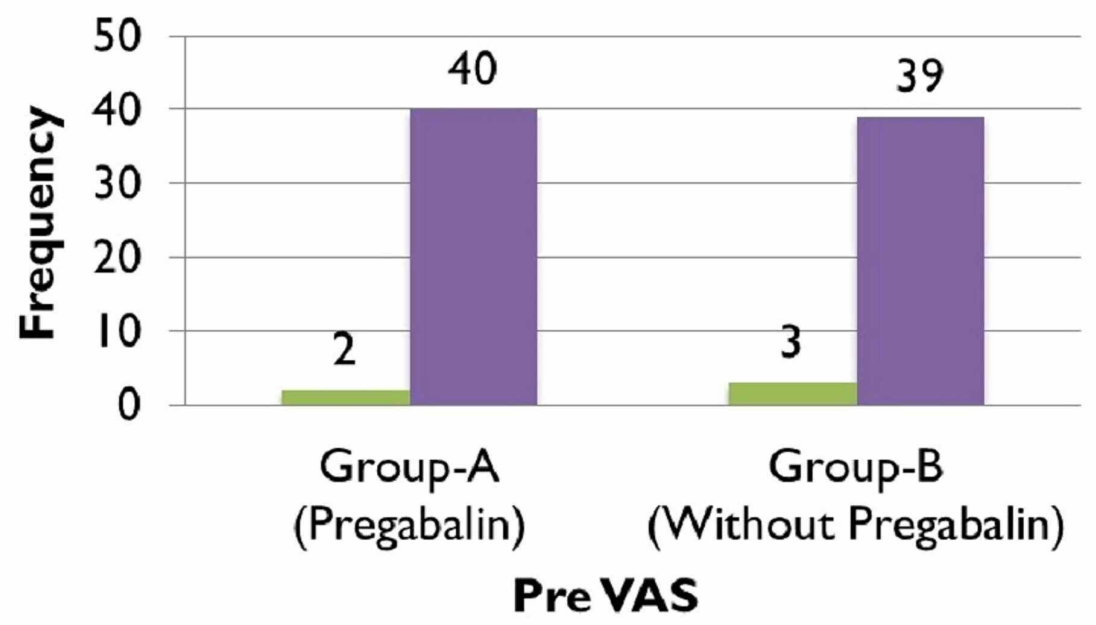

FIGURE 1: Preoperative visual analog scale (VAS) scores with comparison groups

Pearson Chi-square $=0.213, p=0.645 ;{ }^{*}$ Significant at $p<0.05$ 


\section{Cureus}

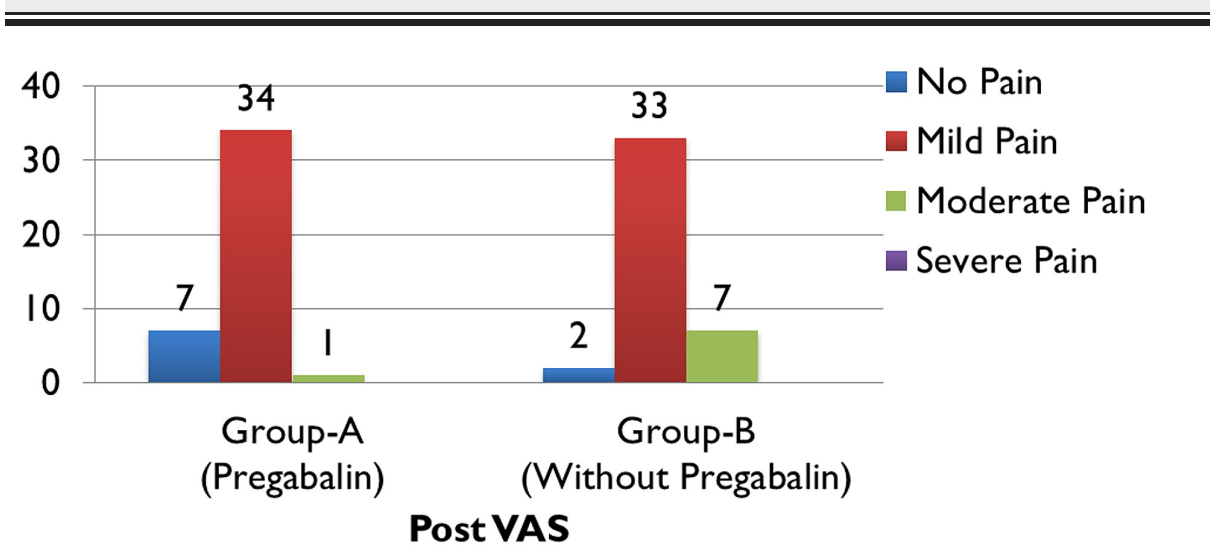

FIGURE 2: Postoperative visual analog scale (VAS) score with comparison groups

Pearson Chi-square $=7.293, p=0.026^{\star} ;{ }^{*}$ Significant at $p<0.05$

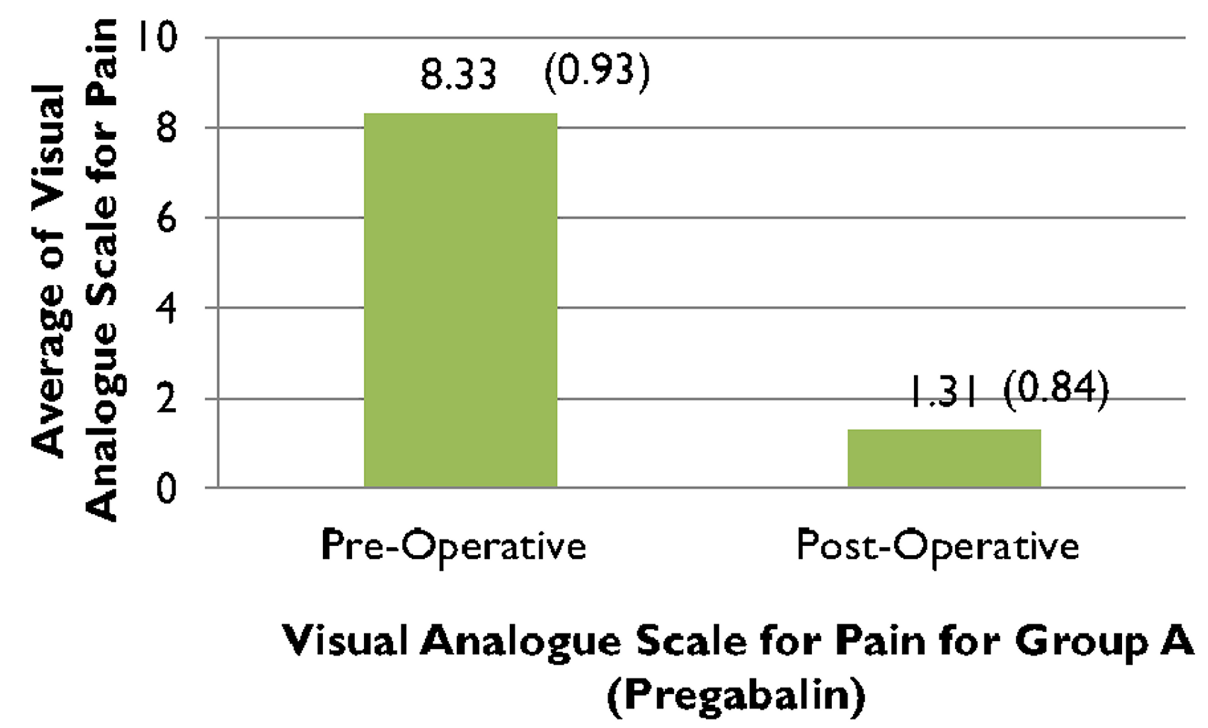

FIGURE 3: Comparison between preoperative and postoperative visual analog scale (VAS) scores for pain for group-A

Paired t-test $=52.369, \mathrm{df}=41 \mathrm{p}<0.0001^{*}$ 


\section{Cureus}

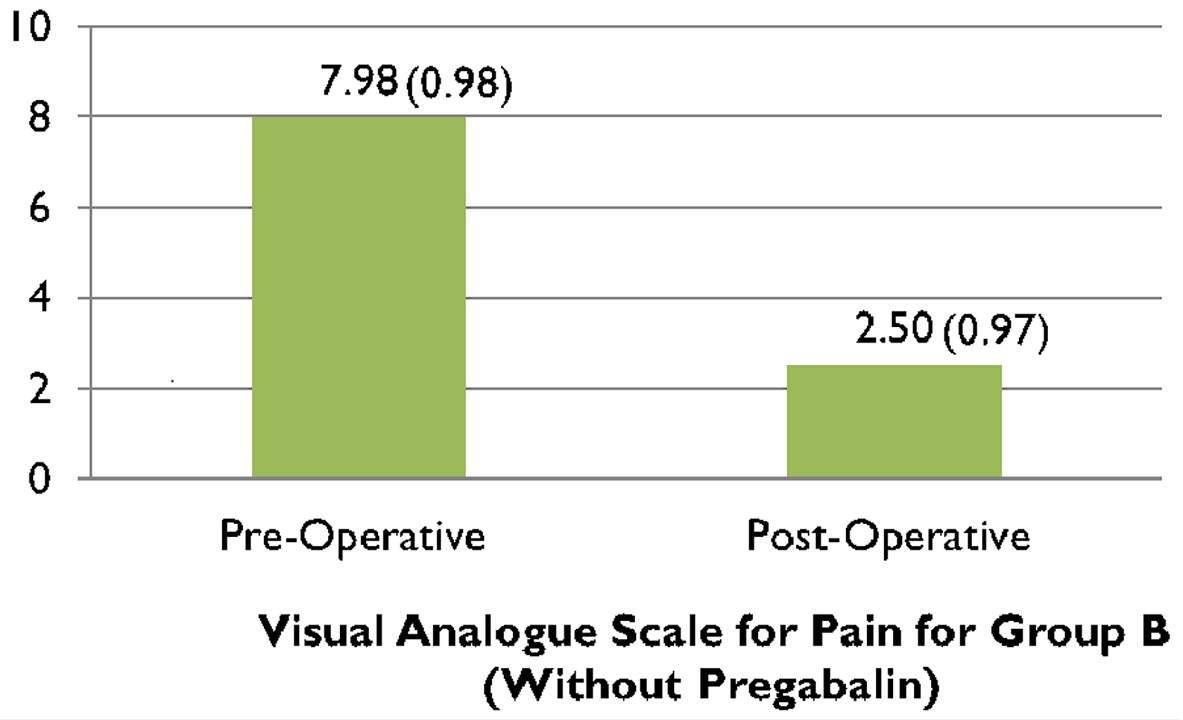

FIGURE 4: Comparison between preoperative and postoperative visual analog scale (VAS) scores for pain for group-B

Paired t-test $=24.725, \mathrm{df}=41 \mathrm{p}<0.0001^{*} ;{ }^{*}$ Significant at $\mathrm{p}<0.05$

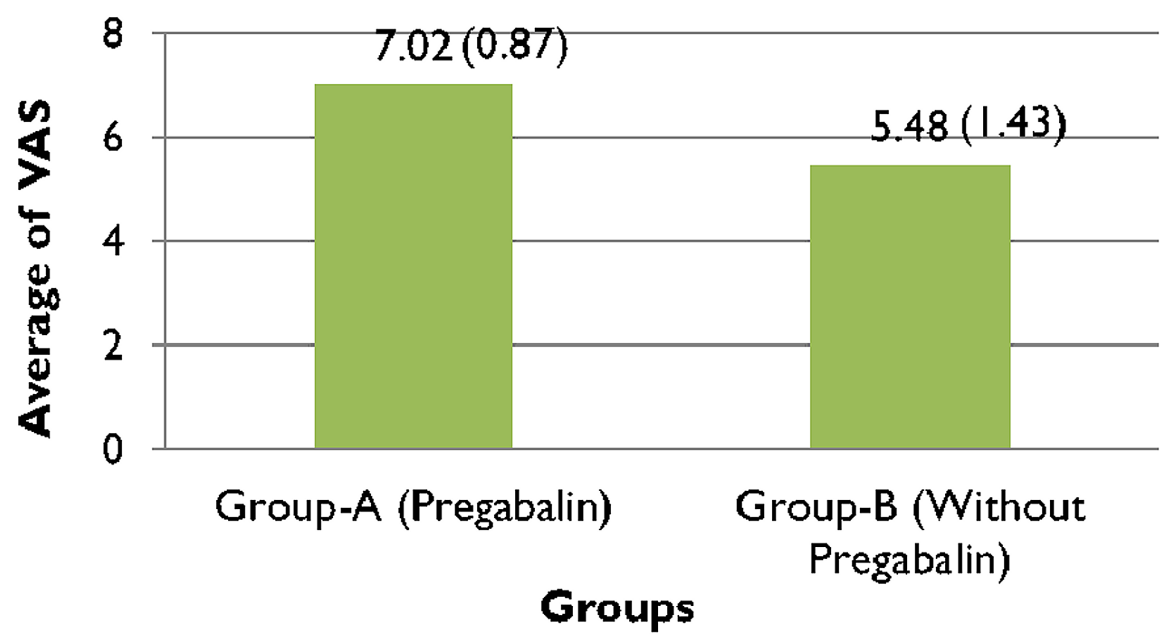

FIGURE 5: Average visual analog scale (VAS) scores comparisons between group-A and group-B

Independent Sample t-test $=5.977, \mathrm{df}=67 \mathrm{p}<0.0001^{*} ;{ }^{*}$ Significant at $\mathrm{p}<0.05$

Pain relief was almost comparable in both genders, with almost $83 \%(n=29)$ of males enduring mild pain and only $8 \%(n=3)$ enduring moderate pain postoperatively, while $77 \%(n=38)$ of females experienced mild pain and almost $10 \%(n=5)$ of them feeling moderate pain. Neither gender reported severe pain postoperatively (Figure 6). For males, the VAS equaled 8.29 preoperatively which considerably dropped to 1.89 postoperatively. Females reported an almost similar score, with their preoperative VAS equaling 8.06 and their postoperative score being 1.92, shown in Figure 7. Figure 8 demonstrates the average VAS for both genders was almost comparable, with the scores being 6.40 and 6.14 for males and females respectively. 


\section{Cureus}
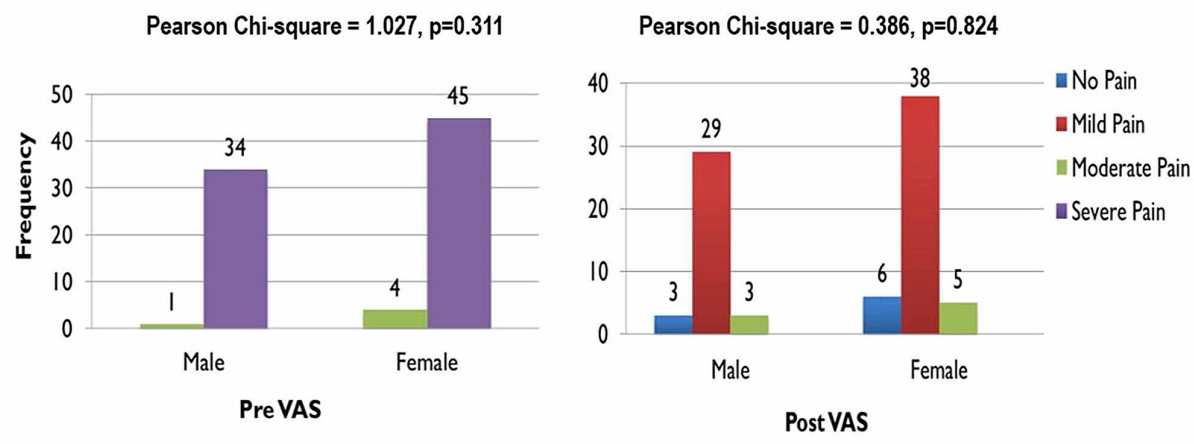

FIGURE 6: Gender wise comparison between pre and postoperative visual analog scale (VAS) scores

Significant at $p<0.05$
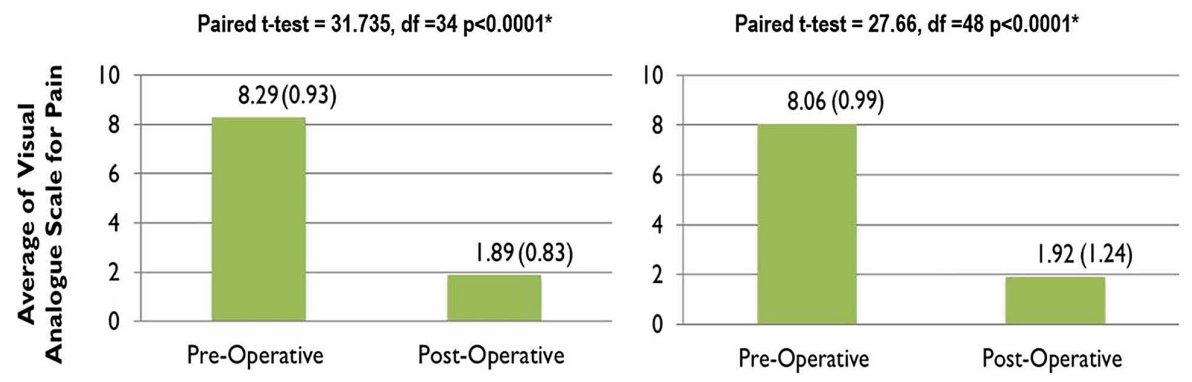

Visual Analogue Scale for Pain for Male

Visual Analogue Scale for Pain for Female

* Significant at $p<0.05$

FIGURE 7: Comparison between pre \& postoperative visual analog scale (VAS) scores in both males and females

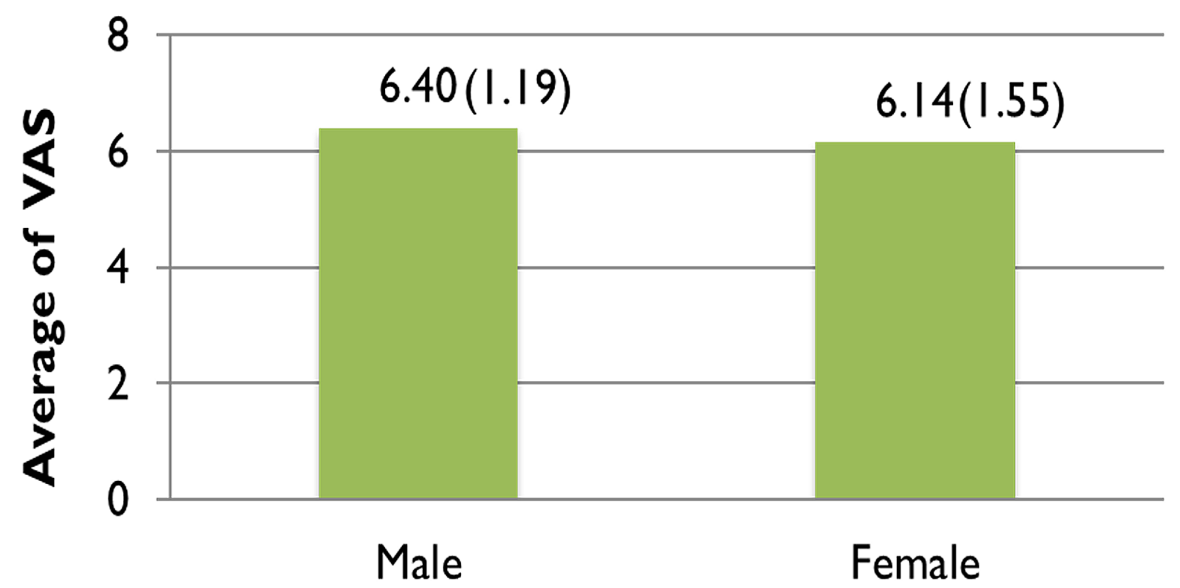

\section{Gender}

FIGURE 8: Average of visual analog scale (VAS) scores in males and females

Independent Sample t-test $=0.812, \mathrm{df}=82 \mathrm{p}=0.414$ 


\section{Cureus}

The average RMS for group A, who were administered pregabalin pre and postoperatively, was 16.43 preoperatively, which considerably reduced to 6.90 postoperatively. However, for group B the scores did not show a great improvement, with the score being 16.19 preoperatively going down to only 9.74 postoperatively (Figure 9).

t-test $=0.404, p=0.687$

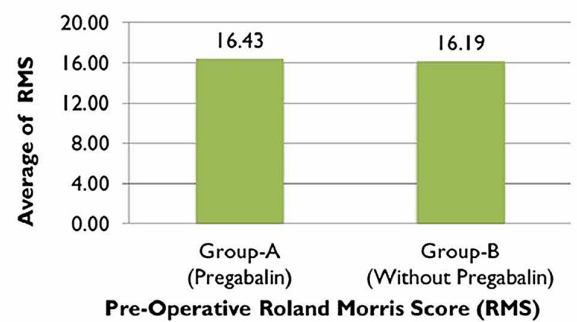

t-test $=-0.5766, p<0.0001$

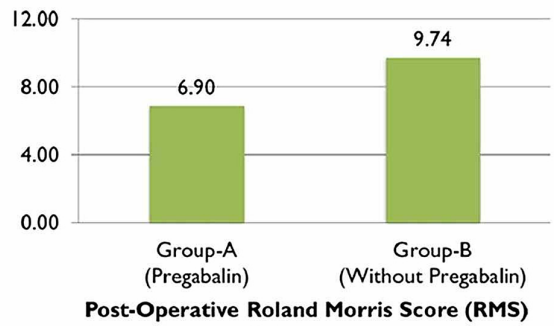

* Significant at $p<0.05$

FIGURE 9: Comparisons of preoperative and postoperative RolandMorris scores

There was also a notable difference between the two groups for PRN consumption of analgesics postoperatively, which, for group A was only $10 \%(n=4)$ while that of group B was $90 \%(n=38)$ as illustrated in (Figure 10), thereby aiding in confirming the hypothesis.

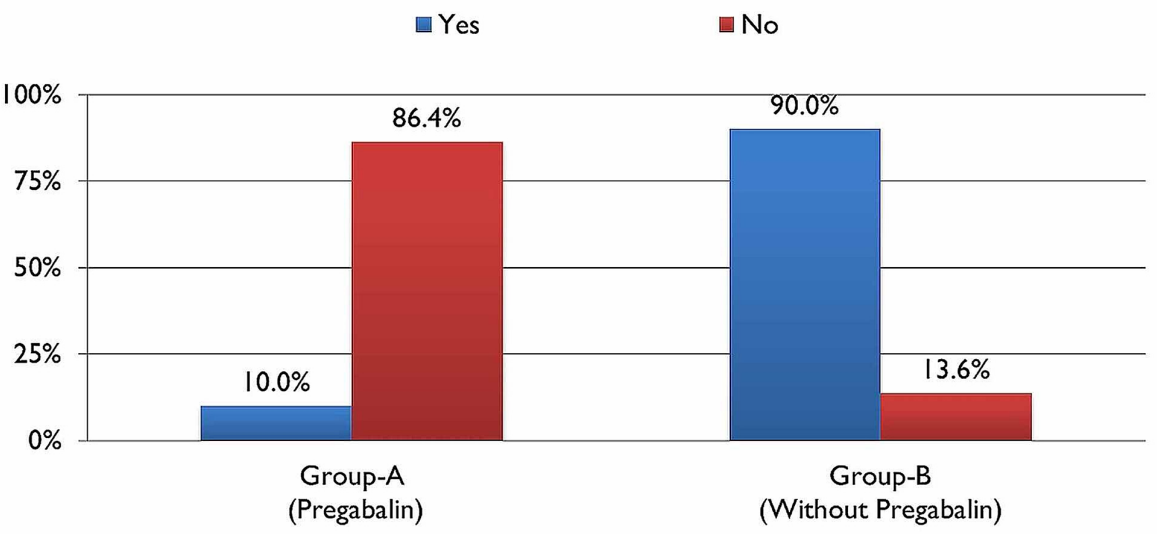

PRN Postoperative Analgesia Consumption

\section{FIGURE 10: PRN (Pro Re Nata) postoperative analgesia consumption with comparison groups}

\section{Discussion}

The study revealed that the pre and postoperative administration of the combination of pregabalin and regular analgesia resulted in improved pain control compared with regular analgesic drug alone after singlelevel microdiscectomy for prolapsed intervertebral lumbar disc.

The combination of the two drugs produced a significant reduction in pain scores (VAS score: 7.02; statistical significance of: 0.87 , p value: $<0.0001 *$ ) compared to regular analgesia (VAS score 5.48 ; statistical significance of: 1.43 , $p$-value: $<0.0001^{*}$ ) (significance of $p=<0.05$ ). 
It has been noted that pregabalin has been more effective in providing analgesic effects for neuropathic pain, [14,20] such as in postherpetic neuralgia [21], lower limb orthopedic surgery [22], and postLichtenstein herniorrhaphic pain [23].

Pregabalin exhibits its anticonvulsant, analgesic, and anxiolytic activity by binding to the ligand for the alpha-2-delta subunit of voltage-gated calcium channels in the central nervous system which thereby reduces depolarization-induced calcium influx along with a modification in the release of excitatory neurotransmitters. Pregabalin inhibits the release of neurotransmitters including glutamate, noradrenaline, calcitonin gene-related peptide, and substance $\mathrm{P}$ [24]. The reduction in a combination of several neurotransmitters is responsible for decreased pain scores as observed in our study.

The extensive and rapid absorption of pregabalin is proportional to the dose. Maximal plasma concentration is attained in approximately one hour while steady-state is accomplished within 24-48 hours which shows that the observed onset of efficacy for pregabalin, is as early as day two in most clinical trials. The mean elimination half-life is calculated to be around 6.3 hours. These aforementioned factors predict a doseresponse relationship in clinical practice, thereby allowing an effective starting dose of $150 \mathrm{mg} /$ day to be administered without any need for titration [14].

In our study, we administered pregabalin to our patients preoperatively, approximately (24) hours before the surgery. As a result, it is expected that the peak plasma concentration for pregabalin was achieved.

We also found in our study that preoperative pregabalin decreased usage of postoperative opioid use for pain management significantly. (P-value $<0.0001$ ). Our results also correspond with a randomized controlled study which concluded that in the first 48 hours post-procedure, the number of doses needed for rescue analgesia, and total required dose of morphine, were significantly less $(p=<0.0001)$ [25].

A study conducted in Denmark on women undergoing hysterectomy concluded that by administering spinal or epidural anesthesia, there is blockade of the central impulse traffic, that produces a protective effect towards the development of chronic pain, eventually preventing extreme postoperative discomfort [26,27]. There is also data supporting the use of spinal anesthesia over general anesthesia, in gynecological procedures such as cesarean sections, for reducing postoperative pain $[27,28]$.

In another study conducted in March 2017 by Liu B, et al. it was concluded that when patients undergoing spinal surgery, were administered gabapentinoids preoperatively, they had a significant reduction in postoperative pain, total consumption of morphine, and morphine-related complications postoperatively [29]. Gabapentin has also shown a rapid response in multimodal analgesia [30].

\section{Conclusions}

The use of pregabalin in addition to the routine analgesia has better control of postoperative neuropathic pain in patients with single-level microdiscectomy compared to the patients who are receiving only routine analgesia. Other factors like cost, dose, side effects, and frequency should also be considered.

\section{Additional Information \\ Disclosures}

Human subjects: Consent was obtained or waived by all participants in this study. Ziauddin University Ethics Review committee issued approval 1090519SRORT. Protocol Title: Role of pregabalin to decrease post-operative pain in mirodiscectomy: A randomized controlled trial On behalf of ERC, I'm pleased to inform you that your research proposal titled " Role of pregabalin to decrease post-operative pain in mirodiscectomy: A randomized controlled trial " has been approved. Animal subjects: All authors have confirmed that this study did not involve animal subjects or tissue. Conflicts of interest: In compliance with the ICMJE uniform disclosure form, all authors declare the following: Payment/services info: All authors have declared that no financial support was received from any organization for the submitted work. Financial relationships: All authors have declared that they have no financial relationships at present or within the previous three years with any organizations that might have an interest in the submitted work. Other relationships: All authors have declared that there are no other relationships or activities that could appear to have influenced the submitted work.

\section{References}

1. Kehlet H, Dahl JB: The value of "multimodal" or "balanced analgesia" in postoperative pain treatment . Anesth Analg. 1997, 77:1048-1056. 10.1213/00000539-199311000-00030

2. Helander EM, Menard BL, Harmon CM, et al.: Multimodal analgesia, current concepts, and acute pain considerations. Anesth Analg. 2017, 21:1048-56. 10.1007/s11916-017-0607-y

3. Kehlet H, Wilmore DW: Multimodal strategies to improve surgical outcome. Am J Surg. 2002, 183:630-41. 10.1016/s0002-9610(02)00866-8

4. Altiparmak B, Güzel Ç, Gümüş Demirbilek S: Comparison of preoperative administration of pregabalin and duloxetine on cognitive functions and pain management after spinal surgery: a randomized, double-blind, 
placebo-controlled study. Clin J Pain. 2018, 34:1114-1120. 10.1097/AJP.0000000000000640

5. Elvir-Lazo OL, White PF: The role of multimodal analgesia in pain management after ambulatory surgery . Curr Opin Anaesthesiol. 2010, 23:697-703. 10.1097/ACO.0b013e32833fad0a

6. Wick EC, Grant MC, Wu CL: Postoperative multimodal analgesia pain management with nonopioid analgesics and techniques: a review. JAMA Surg. 2017, 152:691-697. 10.1001/jamasurg.2017.0898.

7. Apfelbaum JL, Chen C, Mehta SS, Gan TJ: Postoperative pain experience: results from a national survey suggest postoperative pain continues to be undermanaged. Anesth Analg. 2003, 97:534-40. 10.1213/01.ane.0000068822.10113.9e

8. Tan M, Law LS, Gan TJ: Optimizing pain management to facilitate enhanced recovery after surgery pathways. Can J Anaesth. 2015, 62:203-18. 10.1007/s12630-014-0275-x

9. Lavand'homme P, Steyaert A: Opioid-free anesthesia opioid side effects: tolerance and hyperalgesia . Best Pract Res Clin Anaesthesiol. 2017, 31:487-498. 10.1016/j.bpa.2017.05.003

10. Kumar RJ, Menon KV, Ranjith TC: Use of epidural analgesia for pain management after major spinal surgery. J Orthop Surg. 2003, 11:67-72. 10.1177/230949900301100114

11. Mathiesen O, Dahl B, Thomsen BA, Kitter B, Sonne N, Dahl JB, Kehlet H: A comprehensive multimodal pain treatment reduces opioid consumption after multilevel spine surgery. Eur Spine J. 2013, 22:2089-96. 10.1007/s00586-013-2826-1

12. Lam DMH, Choi SW, Wong SSC, Irwin MG, Cheung CW: Efficacy of pregabalin in acute postoperative pain under different surgical categories: a meta-analysis. Medicine (Baltimore). 2015, 94:e1944. 10.1097/MD.0000000000001944.

13. Tesfaye S: Advances in the management of diabetic peripheral neuropathy . Curr Opin Support Palliat Care. 2009, 3:136-43. 10.1097/SPC.0b013e32832b7df5.

14. Ben-Menachem E: Pregabalin pharmacology and its relevance to clinical practice. Epilepsia. 2004, 45:13-18. 10.1111/j.0013-9580.2004.455003.x

15. Sabatowski R, Gálvez R, Cherry DA, Jacquot F, Vincent E, Maisonobe P, Versavel M: Pregabalin reduces pain and improves sleep and mood disturbances in patients with post-herpetic neuralgia: results of a randomised, placebo-controlled clinical trial. Pain. 2004, 109:26-35. 10.1016/j.pain.2004.01.001.

16. Feltner DE, Crockatt JG, Dubovsky SJ, et al.: A randomized, double-blind, placebo-controlled, fixed-dose, multicenter study of pregabalin in patients with generalized anxiety disorder. J Clin Psychopharmacol. 2003, 23:240-249. 10.1097/01.jcp.0000084032.22282.ff

17. Pande AC, Feltner DE, Jefferson JW, et al.: Efficacy of the novel anxiolytic pregabalin in social anxiety disorder: a placebo-controlled, multicenter study. Journal of Clinical Psychopharmacology. 2004, 24:141149. 10.1097/01.jcp.0000117423.05703.e7

18. Qadeer. M, Waqas M, Rashid MJ, Enam A, Sharif S, Murtaza G: Preventive gabapentin versus pregabalin to decrease postoperative pain after lumbar microdiscectomy: a randomized controlled trial. Asian Spine Journal. 2017, 11:93-98.

19. Buvanendran A, Kroin JS, Kari M, Tuman KJ: Can a single dose of $300 \mathrm{mg}$ of pregabalin reach acute antihyperalgesic levels in the central nervous system?. BMJ Regional Anesthesia \& Pain Medicine. 2010, 35:535-538. 10.1097/AAP.0b013e3181fa6b7a

20. Lesser H, Sharma U, LaMoreaux L, Poole RM: Pregabalin relieves symptoms of painful diabetic neuropathy: a randomized controlled trial. Neurology. 2004, 63:2104-10. 10.1212/01.wnl.0000145767.36287.a1.

21. Parsons B, Pan X, Xie L, Chen Y, Ortiz M, Whalen E: Comparison of the efficacy and safety of pregabalin for postherpetic neuralgia in Chinese and international patients. J Pain Res. 2018, 11:1699-1708. 10.2147/JPR.S157856

22. Akhavanakbari G, Entezariasl M, Isazadehfar K, Mirzarahimi T: The effects of oral pregabalin on postoperative pain of lower limb orthopedic surgery: a double-blind, placebo-controlled trial. Perspect Clin Res. 2013, 4:165-8. 10.4103/2229-3485.115376

23. Moghimi MH, Hosseini SN, Salehi V, Kamali K: The effects of oral pregabalin on post-Lichtenstein herniorrhaphic pain: a randomized clinical trial. J Basic Clin Physiol Pharmacol. 2018, 29:321-327. 10.1515/jbcpp-2016-0196

24. Dooley DJ, Donovan CM, Pugsley TA: Stimulus-dependent modulation of [3h] norepinephrine release from rat neocortical slices by gabapentin and pregabalin. J. Pharmacol Exp Ther. 2000, 295:1086-1093.

25. Abdelfattah AAM, Rizk F, Hawash N, Hanafy A, El-Kalla F, Abd-Elsalam S: Randomized trial of preoperative administration of oral pregabalin for postoperative analgesia in patients scheduled for radiofrequency ablation of focal lesions in the liver. Int J Hyperthermia. 2018, 34:1367-1371. 10.1080/02656736.2018.1424946

26. Brandsborg B: Pain following hysterectomy: epidemiological and clinical aspects . Dan Med J. 2012, 59:B4374.

27. Pogatzki-Zahn EM, Segelcke D, Schug SA: Postoperative pain-from mechanisms to treatment . Pain Rep. 2017, 2:e588. 10.1097/PR9.0000000000000588

28. Nikolajsen L, Sørensen HC, Jensen TS, Kehlet H: Chronic pain following caesarean section. Acta Anaesthesiol Scand. 2004, 48:111-6. 10.1111/j.1399-6576.2004.00271.x

29. Liu B, Liu R, Wang L: A meta-analysis of the preoperative use of gabapentinoids for the treatment of acute postoperative pain following spinal surgery. Medicine (Baltimore). 2017, 96:e8031. 10.1097/MD.0000000000008031

30. Buvanendran A, Kroin JS: Multimodal analgesia for controlling acute postoperative pain . Curr Opin Anaesthesiol. 2009, 22:588-93. 10.1097/ACO.0b013e328330373a. 